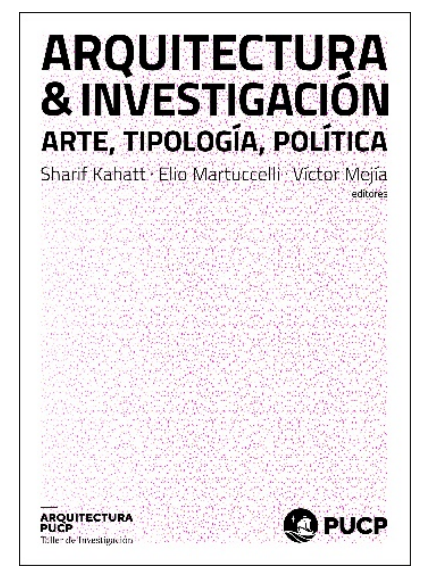

Arquitectura \& Investigación

Arte, tipología, política

ISBN 978-612-47555-5-2

Ideales versus realidades. Tres ciudades universitarias en Lima a mediados del siglo XX

Valeria Takano Reyes

Para citar este artículo: Takano R., V. (2021). Ideales versus realidades. Tres ciudades universitarias en Lima a mediados del siglo XX. En S. Kahatt, E. Martuccelli y V. Mejía (Eds.), Arquitectura \& Investigación. Arte, tipología, política (p. 139.156). Pontificia Universidad Católica del Perú.

DOI: $\underline{10.18800 / 978-612-47555-5-2.007}$

Para acceder a este artículo: https://doi.org/10.18800/978-612-47555-5-2.007

\title{
ARQUITECTURA PUCP
}




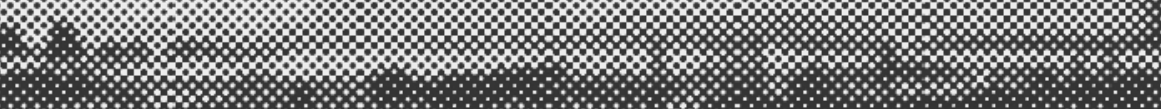
mo

\section{\%om} \%om \%क\% 3\% \%

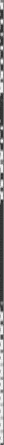

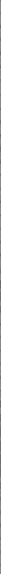




\title{
IDEALES VERSUS REALIDADES Tres ciudades universitarias en Lima a mediados del siglo XX
}

\author{
Valeria Takano Reyes
}

\begin{abstract}
Resumen
El presente artículo aborda el estudio de las ciudades universitarias en Lima a mediados del siglo XX; en específico, tres casos de estudio importantes para la idiosincrasia y configuración de la ciudad: la Universidad Nacional Mayor de San Marcos (UNMSM), la Universidad Nacional de Ingeniería (UNI) y la Universidad Nacional Agraria La Molina (UNALM). La ciudad universitaria como proyecto moderno simboliza el anhelo por el desarrollo de una ciudad en crecimiento como Lima. La identificación de sus modelos urbanísticos y arquitectónicos, situados en un lugar de implantación determinado según condicionantes políticos, económicos y culturales, cada uno particular, revaloriza el concepto de «ciudad universitaria» en la historia del modernismo en el Perú. Así también, da pie a la reflexión sobre el papel de la ciudad universitaria y sus implicancias en la estructura urbana de la ciudad.
\end{abstract}

Palabras clave: ciudad universitaria, proyecto urbano, movimiento moderno, Estado-nación, Lima.

\section{Abstract}

This article addresses the study of university campuses in Lima in the mid-twentieth century, specifically three important case-studies for the characteristics and formation of the city: The «National University of San Marcos» (UNMSM), the «National University of Engineering» (UNI), and the «National Agrarian University La Molina» (UNALM). The university campus as a modern project symbolizes the desire for the modernization and development of a growing city like Lima. By identifying its urban and architectural types - whose location was determined by different political, economic, and

Valeria Takano Reyes

Estudiante de la Facultad de Arquitectura y Urbanismo PUCP. Fue practicante pre profesional en la Dirección de Infraestructura PUCP (Sección de Proyectos). En 2019 fue Vicepresidenta del Centro Federado de Arquitectura y Urbanismo PUCP. Desarrolló el presente artículo durante el ciclo académico 2019-2. cultural conditions, the concept of «university campus» is reassessed in $\mathrm{Pe}$ ru's modernism history. Likewise, this study reconsiders the role of university campuses and their effects in the urban structure of the city.

Keywords: university campus, urban project, Modern Movement, Nation State, Lima. 


\title{
IDEALES VERSUS REALIDADES \\ Tres ciudades universitarias en Lima a mediados del siglo XX
}

\author{
Valeria Takano Reyes
}

\section{Crecimiento en Lima: masas y territorio}

El proceso de expansión de la ciudad de Lima hasta mediados del siglo XX se inició en la década de 1920, con una población de 170000 habitantes, de los cuales 60000 eran de origen provinciano (Garfias 2009: 124). Ya para la década de 1940, terminada la Segunda Guerra Mundial, se respiraba un clima de democracia, crecimiento económico y estabilidad política que permitió el desarrollo urbano de la ciudad, así como del oficio arquitectónico. El trazado de grandes ejes, como la avenida Brasil, la avenida Leguía (hoy Arequipa) y el Paseo de la República, que conducía al sur, propiciaron la conformación de nuevos distritos y centros de la ciudad. Siguiendo a Wiley Ludeña,

La década del cuarenta resulta, ciertamente, una década de muchas primeras veces para el urbanismo limeño. Apareció por primera vez como demanda profesional y pública el tema del Plan Moderno y la necesidad de regular el crecimiento de la ciudad sobre la base de una serie de orientaciones y parámetros de desarrollo (2001: 247).

El gobierno de José Luis Bustamante y Rivero (1945-1948) sentó las bases para nuevos proyectos de renovación en el ámbito educativo y en el urbanístico, con la creación de la Corporación Nacional de la Vivienda (CNV, 1946) y la Oficina Nacional de Planeamiento y Urbanismo (ONPU, 1946). El Instituto de Urbanismo se creó posteriormente, en 1951. Sin embargo, la implementación de servicios y de infraestructura también significó un aumento exponencial de la población limeña. Entre 1940 y 1961 la zona metropolitana pasó de 645000 a 1845000 habitantes; en términos espaciales, creció el área periférica de Magdalena, Miraflores, Lince, San Isidro, La Victoria y Surco, mucho más que la propia área central (Gutiérrez 1983: 683). En la década de 1950, bajo el gobierno de Manuel A. Odría, las migra-

1 Manuel A. Odría ejerció el poder durante ocho años — de ahí la designación «Ochenio de Odría»-, tras el golpe de Estado contra el presidente Bustamante y Rivero en 1948 , en una etapa de fuerte militarismo. ciones dieron lugar a nuevas barriadas limeñas. ${ }^{1}$ Las primeras, Mendocita y San Cosme, seguidas de tres grandes núcleos: San Martín de Porres (1952), Pampa de Comas (1958) y El Ermitaño (1962) (Gutiérrez 1983: 685).

Es importante adelantar que las necesidades de las grandes masas estudiantiles propiciaron la búsqueda de nuevos fundos de gran amplitud 
en la periferia de Lima, que pudieran absorber la demanda. No es de extrañar que, luego de la construcción de las principales ciudades universitarias de la época, se iniciara el crecimiento de la ciudad en dichas zonas, gracias a la valorización de los terrenos adyacentes a estos fundos.

\section{Las ciudades universitarias en Latinoamérica y el Perú}

En palabras del urbanista francés Pierre Merlin, «la universidad no siempre ha sido urbana» (2006: 183). El origen de las ciudades universitarias se remonta a inicios del siglo XIX, en un contexto en el que, debido a la Revolución Industrial, las universidades en bloque resultan insuficientes para las nuevas masas estudiantiles. Según el historiador Antonio Bonet, tanto las universidades estadounidenses como las europeas se plantearon la polémica de la implantación de la ciudad universitaria como «un conjunto compacto de edificios o disperso en un área libre» (2014: 29). Sin embargo, no fue sino hasta la segunda mitad del siglo XX cuando apareció la especialización americana del campus planner y la idea predominante de que el campus constituía la ciudad (Merlin 2006: 187). Es así como se fue diversificando la tipología de las ciudades universitarias con arquitecturas heterogéneas, en la búsqueda de un carácter nacional particular. Coincide con ello la influencia del Movimiento Moderno, que sintetiza en la Carta de Atenas los principios urbanísticos modernos por los que se regiría el planeamiento de campus universitarios ubicados territorialmente en espacios dispersos, potenciales para una libre composición urbanística. ${ }^{2}$

En Latinoamérica no fue diferente. Como menciona Carlos Fino, entre 1935 y 1960 surgió un impulso continental por construir ciudades universitarias con la intención de imitar los campus estadounidenses y por la necesidad educativa de las naciones «modernas» de adaptar un modelo democrático de carácter liberal (2018). Parte del reconocimiento del modelo arquitectónico y urbanístico de las ciudades universitarias en Latinoamérica reside en la integración de la modernidad y el respeto a la tradición de cada nación. Tan es así que la Universidad Nacional Autónoma de México, diseñada por Mario Pani y Enrique del Moral, y la Universidad de Caracas, por Carlos Raúl Villanueva, son consideradas Patrimonio Cultural de la Unesco. El modelo de la ciudad universitaria fue un símbolo de modernidad y desarrollo del nuevo Estado-nación: los principios de la autonomía, la democracia y la sociedad civil se reflejaban en una organización racional de edificios de excelente calidad, con espacios públicos para todos sus «ciudadanos» estudiantiles.

En la década de 1940 las ciudades universitarias tuvieron mayor

2 Ya desde el Congreso Internacional de Arquitectura Moderna (CIAM) de La Sarraz (1928) se impulsó el «espíritu nuevo» lecorbusiano, a partir del quiebre entre el viejo orden y los avances científicos y tecnológicos de la época (Gutiérrez y Gutiérrez 2012: 2). acogida en el Perú, a partir de la publicación, en la revista El Arquitecto Peruano, de una primera nota sobre el tema, titulada «Una ciudad universitaria peruana» (enero de $1941, \mathrm{n}^{\circ}{ }^{\circ}$ 42), en la que se hacía una crítica al gobierno y a la tradición como principales enemigos de la modernización. Dos años después en la misma revista se publicaron una serie de planes maestros de ciudades universitarias latinoamericanas, que 
servirían de modelo para el planteamiento de nuestros campus: de Río de Janeiro, diseñado por Le Corbusier y Pierre Jeanneret (1936); de Bogotá, por Leopoldo Rother y Erich Lange (1939); y de Buenos Aires, por la Comisión de Urbanismo de la Sociedad Central de Arquitectos (SCA, 1939), entre otros. En resumen, la revista resalta su incesante preocupación por el retraso del país en comparación con las naciones vecinas (El Arquitecto Peruano, n. ${ }^{\circ} 71,1943$ ).

El concepto de universidad como «ciudad en miniatura», propio del Movimiento Moderno, llegó a Lima mediante el impulso de personajes ilustres del ámbito académico tanto de la Universidad Nacional Mayor de San Marcos como de la Escuela de Ingeniería. En el resto del país, las universidades mantenían un carácter colonial propio del siglo XVIII — como en la Universidad de San Cristóbal de Huamanga (Ayacucho) y la Universidad de San Antonio Abad (Cusco)_- Ya en el siglo XIX, el modelo republicano nace con la Universidad Nacional de Trujillo (jesuitas) y la Universidad Nacional de San Agustín (Arequipa); sin embargo, si bien esta última tuvo una remodelación en 1936, a cargo del arquitecto Héctor Velarde, no formó parte de la tipología de ciudad universitaria basada en principios modernistas. Los casos de estudio se sitúan en Lima, por su singularidad frente al resto del país.

\section{Implantación territorial e intereses sociopolíticos}

La educación pública superior en Lima, hasta mediados del siglo XX, giró alrededor de la Universidad Nacional Mayor de San Marcos (UNMSM), la Escuela Nacional de Ingeniería (ENI, posteriormente UNI) y la Escuela Nacional de Agricultura y Veterinaria (ENAV, posteriormente UNALM). Estos centros, de gran importancia para la sociedad limeña, fueron los espacios de encuentro entre intelectuales nacionales e internacionales de la época, impulsores de la modernización de la educación superior, semejante a la de otras escuelas de Latinoamérica. La importancia de definir las variantes (localización, área, grado de consolidación, usos, etcétera) reside en la identificación del pensamiento característico de cada espacio universitario para la revalorización del campus y los tejidos urbanos que lo rodean (Cunha 2006: 180). En definitiva, los tres casos de estudio ameritan una comparación tipológica en cuanto que cada uno presenta una identidad académica compleja y particular. Siguiendo el orden cronológico, se sugiere una secuencia basada en etapas predeterminadas.

3 La ENI sufrió la constante amenaza de perder el local en favor de la construcción de la basîlica de Santa Rosa o del ensanche de la avenida Tacna, hecho que ocurrió después de 1948, cuando se trasladó al fundo Puente Palo (Rodríguez 1999: 53).

\section{Antecedentes: futuros truncos}

A inicios del siglo $X X$, las tres instituciones se encontraban ubicadas en el área perimetral del centro de Lima: la UNMSM, en la Casona de San Marcos, junto al Parque Universitario; la ENI, en la calle Espíritu Santo, junto al terreno de la Basílica de Santa Rosa; y la ENAV, en el fundo Santa Beatriz, al sur del centro de la ciudad (actualmente, Parque de la Exposición). ${ }^{3}$ Las dos 


\section{FIGURA 1}

Plano del primer anteproyecto de la ciudad universitaria de la Escuela de Ingenieros, entre las avenidas Brasil y Pershing, con un frente de $154 \times 378$ metros (1939), realizado por los arquitectos Ricardo Malachowski, Rafael Marquina y Héctor Velarde. Fuente: Archivo, Proyecto Historia UNI.
4 Luis Felipe Villarán, rector de la UNMSM de 1905 a 1913 hace hincapié en el problema del local universitario: «Este viejo edificio es ya inapropiado para el funcionamiento de la Universidad. [...] las condiciones de un local amplio y apropiado contribuyen a hacer más eficaz la enseñanza [...] y porque San Marcos debe estar al nivel de las universidades extranjeras» (Fabbri 2014: 380).

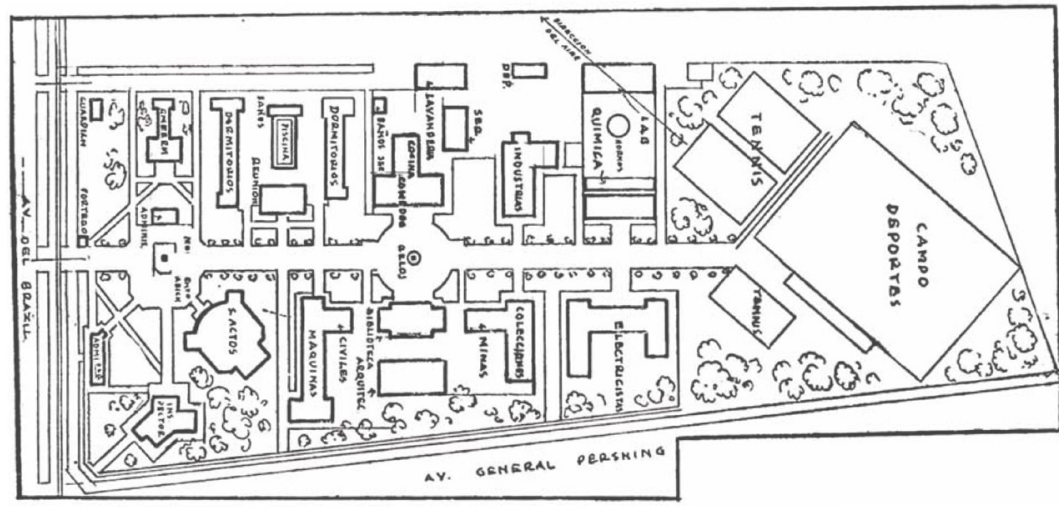

primeras instituciones —y las más populares - tenían el mismo problema de deterioro por la antigüedad de sus locales, así como la imposibilidad de crecer en su perímetro, ya que se encontraban en un tejido consolidado y en pleno desarrollo. Por esta razón se empezó a demandar el traslado a zonas más apropiadas, sin el bullicio y la actividad de la ciudad. ${ }^{4}$ En el caso de la ENI, el problema no solo era la demanda estudiantil, sino también los requerimientos de espacio para laboratorios y gabinetes: su ubicación en la ciudad no era apropiada porque estos expedían humos y hacían mucho ruido (Rodríguez 1999: 53). La ENAV no tuvo estos problemas, ya que recién iniciaba sus funciones en 1902 en el fundo Santa Beatriz, a las afueras del tejido urbano consolidado, en un terreno de 259 hectáreas que le fue obsequiado por el presidente José Balta en 1870 bajo la premisa de que era necesario desarrollar las tierras agrícolas para el progreso del país y las industrias. Para la UNMSM la situación fue peor, ya que sufrió reducciones de rentas del Estado debido a que el gobierno oligárquico civilista prefirió invertir en nuevas escuelas técnicas para el impulso de una sociedad moderna (Garfias 2009: 151). La corriente positivista sanmarquina propuso la integración de escuelas como la ENI a la universidad, basándose en ejemplos norteamericanos en los que se integraban las enseñanzas humanísticas con las técnicas, generando armonía, prestigio y mismas jerarquías (Garfias 2009: 161). Sin embargo, las escuelas mantuvieron su autonomía para conservar sus distribuciones internas en lugar de acoplarlas todas en una «facultad» dentro de la UNMSM.

\section{Segunda etapa: individualismos y fricciones terrenales}

El Oncenio de Leguía (1919-1930) fue una etapa de crisis para las universidades debido al sistema opresivo del gobierno. En la década de 1920 la ENAV sufrió los mayores estragos, por el desinterés del gobierno y sus malas relaciones con este. El fundo Santa Beatriz tuvo varias reducciones, empezando por 67 hectáreas para la avenida Leguía — que conectaría con Miraflores - en 1921, y las 31 hectáreas donadas por el gobierno para la ciudad universitaria de la UNMSM en 1923. Entre 1920 y 1930 las zonas 
aledañas a la avenida Leguía se fueron urbanizando progresivamente, afectando los terrenos de la ENAV y de la UNMSM (Olcese 2002: 146). En consecuencia, la ENAV perdió cerca de 184 hectáreas, por lo que resultaba imposible mantenerse en una zona ya en proceso de urbanización. Así, en 1926 solicitan al Ministerio de Fomento el traslado de la escuela al fundo La Molina, de 200 hectáreas, ubicado en el valle de Ate, fundo que, convenientemente, pertenecía al presidente Leguía (Yepes 1986: 201). ${ }^{5}$ Ese mismo año se contrató al arquitecto Claude Sahut para el diseño del proyecto; las obras se iniciaron y el local abrió sus puertas en 1933. En contraste, para la UNMSM y la ENI la situación empeoró debido a las movilizaciones estudiantiles después de la caída de Leguía. La UNMSM cerró hasta 1935, y en este lapso de tiempo y readaptación el terreno de Santa Beatriz se vendió al doctor Edgardo Rebagliati para la construcción del Hospital del Empleado (Fabbri 2014: 384).

Mientras tanto, la junta de la ENI continuó con las presiones al Ministerio de Fomento, que culminaron en la adquisición del terreno ubicado entre la avenida Brasil y Pershing, ya bajo el gobierno de Benavides, en 1939 (Rodríguez 1999: 55). Los arquitectos Ricardo Malachowski, Rafael Marquina y Héctor Velarde realizaron los planos del anteproyecto, los cuales nunca fueron construidos (figura 1).

5 En el memorial dirigido al presidente de la República se mencionaba «la imprescindible necesidad de reinstalar la Escuela y sus dependencias en un fundo cercano a Lima. Para conseguirlo es indispensable anexarle una explotación agrícola que servirá de centro de aplicación al futuro ingeniero agrónomo» (Olcese 2002: 194)

6 La ley generó eventuales cambios a lo largo de la década de 1940, tales como la independización de la sección veterinaria de la ENAV en 1943, lo que conllevó el cambio de nombre a Escuela Nacional de Agricultura, ENA (Flores 2000: 175). Por otro lado, en el nuevo Estatuto Universitario de 1946 «se contemplaba la necesidad de construir una Ciudad Universitaria en un terreno expropiado para dicho fin» (Meza 2009: 253).

7 En 1960 ya existía un proyecto de transformación de escuela a universidad, a cargo del Consejo Académico de la ENA. En febrero de ese año se promulgó la Ley 13417, que le asigna el nombre de Universidad Nacional Agraria La Molina (UNALM) (Flores 2000: 177).

\section{Tercera etapa: nuevas aspiraciones}

La década de 1940 fue decisiva para la educación superior universitaria. Durante el gobierno de Manuel Prado ocurrieron dos acontecimientos importantes: se aprobó la Ley Orgánica de Educación Pública 9359, 6 con la cual las instituciones se volverían más autónomas; y acaeció el gran terremoto de Lima y maremoto del Callao (López Soria 2005: 10). La ENA fue la más afectada por el desastre natural, ya que los nuevos edificios terminaron inhabitables debido a la baja resistencia de sus suelos. Por ello se trasladó nuevamente al Centro de Lima, al antiguo local y a otros brindados por los jesuitas, hasta que al año siguiente se regularizaron cuatro pabellones (Olcese 2002). No fue sino hasta 1960 cuando se preparó un Plan Maestro para la Ciudad Universitaria de la UNALM, a cargo de arquitectos e ingenieros nacionales e internacionales que conformaron la Oficina para la Planificación de la Ciudad Universitaria. ${ }^{7}$ El proyecto se insertó en un Programa de Modernización, con participación de la Agencia de Desarrollo Internacional de los Estados Unidos de América (Usaid) y la Universidad Estatal de Carolina del Norte (Olcese 2002: 434). En este caso, el proceso y resultado de la ciudad universitaria fue satisfactorio, en la medida en que recibió apoyo tanto económico como académico, gracias a las buenas relaciones entabladas entre el rector Orlando Olcese y las entidades norteamericanas (figura 2).

Regresando a 1945, terminada la Segunda Guerra Mundial el panorama mejoró y, con el gobierno de José Luis Bustamante y Rivero, se plantearon nuevas posibilidades para la creación de las ciudades universitarias. La UNMSM comenzó a buscar en la periferia nuevos fundos de mayor 


\section{FIGURA 2}

Arquitectos Robert Etheredge y Edward Waugh, de la Universidad de Carolina del Norte, muestran la maqueta de la nueva ciudad universitaria de la Universidad Nacional Agraria La Molina al ingeniero Jacobo Zender y al doctor Orlando Olcese. Fuente: Olcese 2002.

\section{FIGURA 3}

Proyecto de la Ciudad Universitaria de 1957 realizado por los arquitectos Alfredo Dammert, Carlos Morales Macchiavello, Gerardo Lecca y el urbanista Luis Dorich. Perspectiva del ingreso principal que da hacia el "Paseo de estudiantes". Fuente: La Ciudad Universitaria de San Marcos y el proyecto de universidad del siglo XX - Martin Fabbri (2014)
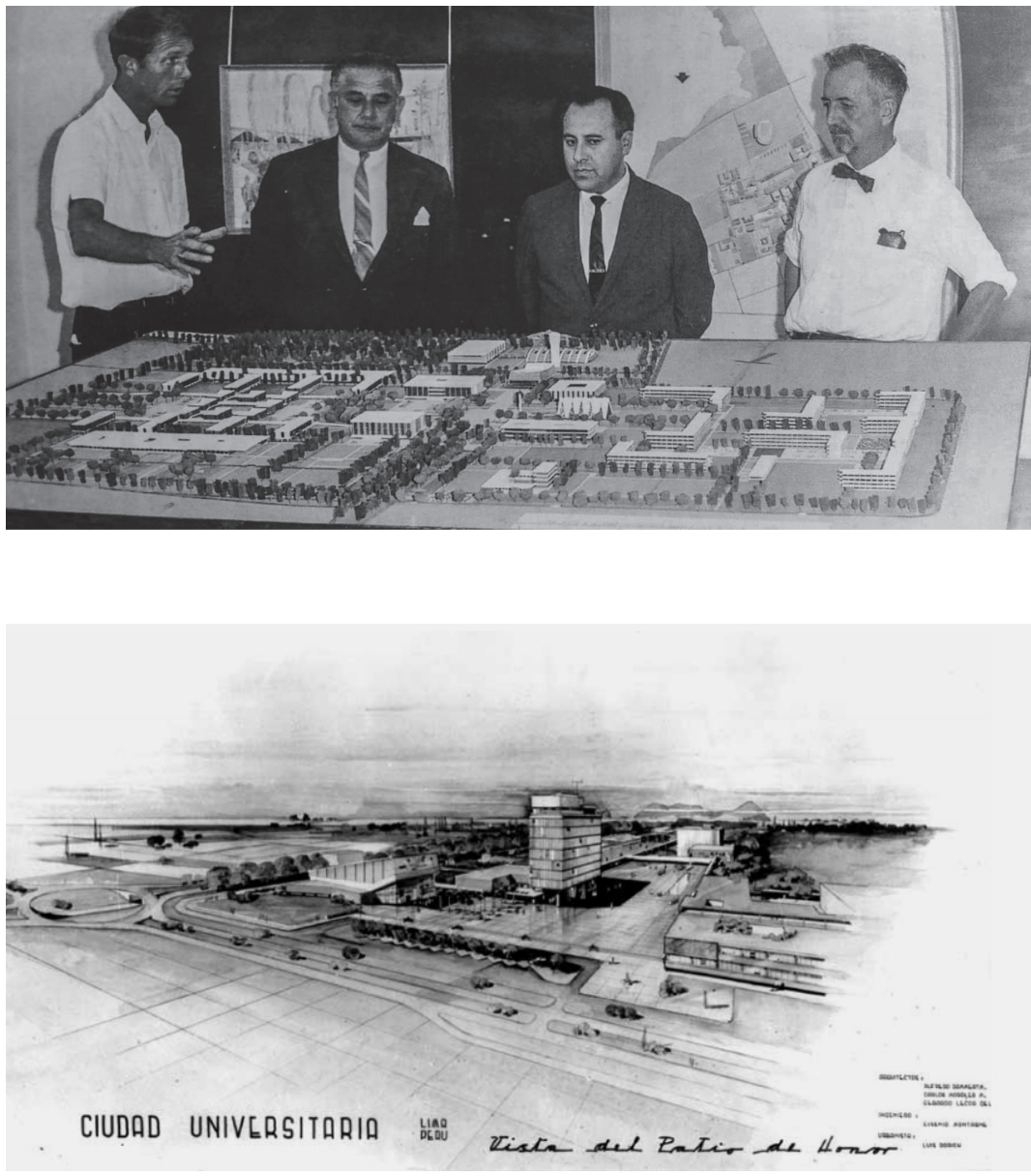


\section{FIGURA 4}

Plano modificado sobre la base del anteproyecto para la Escuela de Ingenieros en el fundo Puente Palo (1944), realizado por la firma Moore \& Hutchins (Nueva York) y los arquitectos peruanos Haaker Fort, Ricardo Valencia y Ricardo Malachowski. Fuente: El Arquitecto Peruano, n. ${ }^{\circ} 82,1944$.

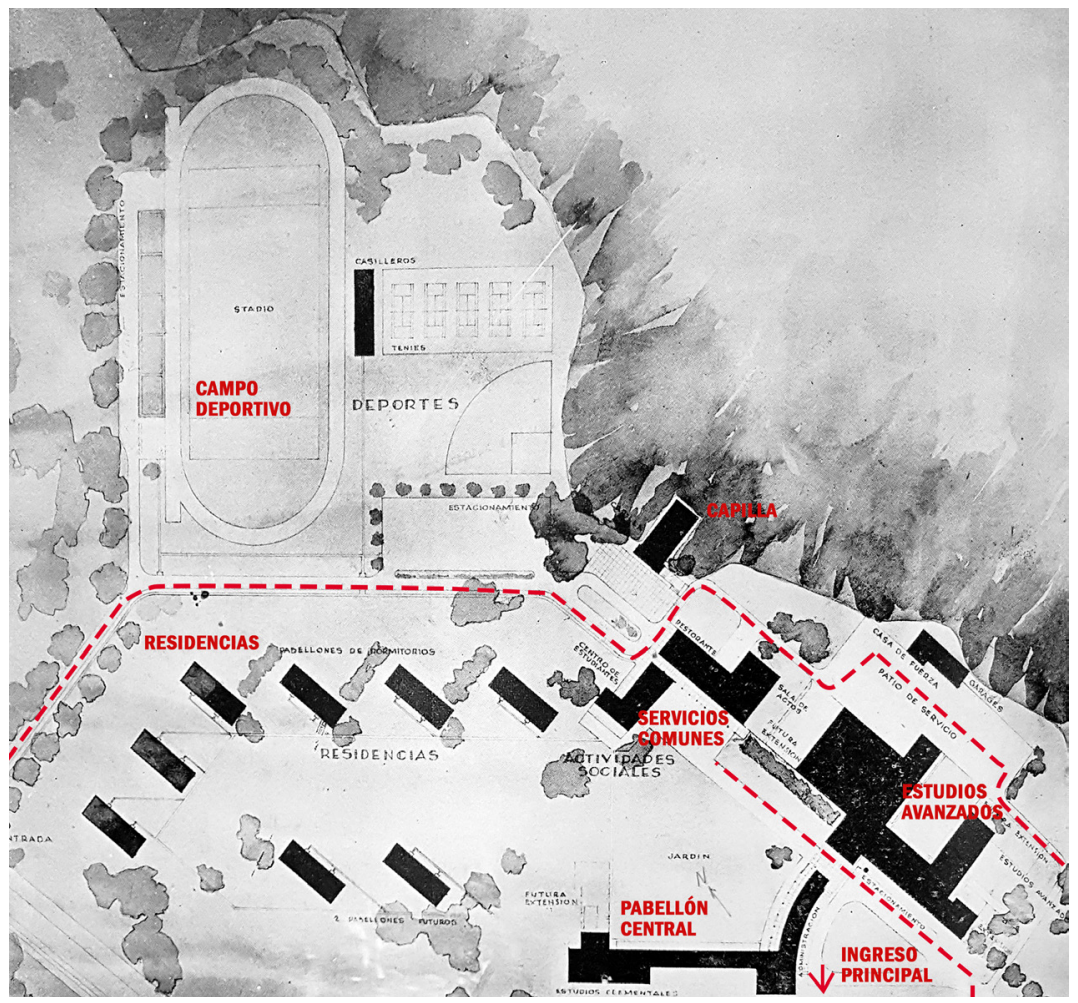


extensión. A partir de las premisas que el rector Luis Alberto Sánchez recogió en el extranjero — las ciudades universitarias de Colombia, Guatemala y Panamá-, este concluyó que «la Ciudad Universitaria no cabía ni cabe en los terrenos [...] [de la avenida Arenales], ni es conveniente su ubicación allí, que sería absolutamente urbana» (Fabbri 2014: 384). Así, se conformó una Comisión de la Ciudad Universitaria para el traslado a los siguientes fundos: terrenos de la urbanización Risso (54 hectáreas); fundo Cueva, Maranga y Pando (268 a más hectáreas); y la zona Vásquez-Salamanca (234 a más hectáreas), cerca de La Molina (Fabbri 2014: 384).

Si bien el fundo Pando estuvo entre los elegidos, este le pertenecía a la Pontificia Universidad Católica del Perú, que lo tenía reservado para la construcción de su ciudad universitaria; y a pesar de que se inició un proceso de expropiación, en 1948 este resultó trunco debido al golpe de Estado de Odría. Ya en 1950, el presidente donó terrenos del Estado ubicados entre las avenidas Venezuela y Benavides (68 hectáreas), destinados en un principio a la construcción del Estadio Nacional, con la condición de que las obras se iniciaran en un plazo máximo de dos años. ${ }^{8}$ La UNMSM no tuvo otro remedio que aceptar la propuesta, bajo un régimen de clientelaje, para lograr la meta de la construcción de la ciudad universitaria en el marco de la celebración de su IV centenario (Meza 2009: 256) (figura 3).

Para la ENI la situación se complicó debido a que, si bien contaba con el terreno donado —entre las avenidas Brasil y Pershing_- las obras no podían comenzar por falta de financiamiento. Además, la Comisión encontró nuevos inconvenientes en la ubicación del terreno en una futura área urbana: la emisión de los gases de los laboratorios, los efectos de la atmósfera marina sobre los instrumentos de la Escuela, el peligro de la división del terreno por calles o avenidas y la venta de lotes para la expansión urbana (Rodríguez 1999: 55). Se planteó, entonces, el traslado de la ciudad universitaria al fundo Puente Palo, ubicado en dirección a Ancón, alejado de la ciudad. Este nuevo terreno presentaba ventajas sobre el anterior. Para comenzar, poseía 36 hectáreas disponibles para las construcciones -el anterior solo tenía 6-, que se podrían comenzar rápidamente gracias a la venta del terreno de la avenida Brasil al general Eloy Ureta, para la construcción del Hospital Militar (1943). Si bien atravesó un largo proceso de consolidación, debido al bajo aporte económico del Estado, la ENI utilizó sus rentas de trabajos ingenieriles para financiar obras y mate-

8 El antiguo terreno para el Estadio Nacional, donado por Odría, incluía el proyecto incompleto y en construcción de dicho estadio, abandonado luego de que se decidiera su traslado al Hipódromo de Lima (Santa Beatriz). Asimismo, la UNMSM debía compartir por decreto, una parte del terreno con el Hospital Naval. Otro inconveniente era la falta de servicios de agua y desagüe, por lo que la universidad debía asumir los gastos de la implementación (Meza 2009: 255). riales; además, la ayuda económica de la Junta Pro Desocupados de Lima permitió el aceleramiento del proyecto (Rodríguez 1999: 60). Para 1948, la ENI dejó de funcionar en Espíritu Santo y se trasladó a Puente Palo. La modernización física de la Escuela conllevó la transformación de la enseñanza técnica de la misma, así como su paso a la categoría de Universidad Nacional de Ingeniería en 1955 (figura 4).

\section{Los campus universitarios y el proyecto moderno}

Una vez que las ciudades universitarias ya se encontraban emplazadas en las afueras de la ciudad, las autoridades de las mismas empezaron la 


\section{FIGURA 5}

Fotografía aérea del fundo ubicado entre las avenidas Venezuela y Benavides (68 hectáreas), en el que se ubicó la Ciudad Universitaria de la UNMSM (1950). Fuente: Fabbri 2014. Imagen editada con información del plano arqueológico publicado en Silva y Paredes 1993.
9 Maranga fue la ciudad principal de la cultura Lima en el valle del Rímac. Del período Intermedio Temprano (200 a. C. a 650 d. C.), destacan las huacas San Marcos y Concha, hoy ubicadas en la Ciudad Universitaria de la UNMSM.

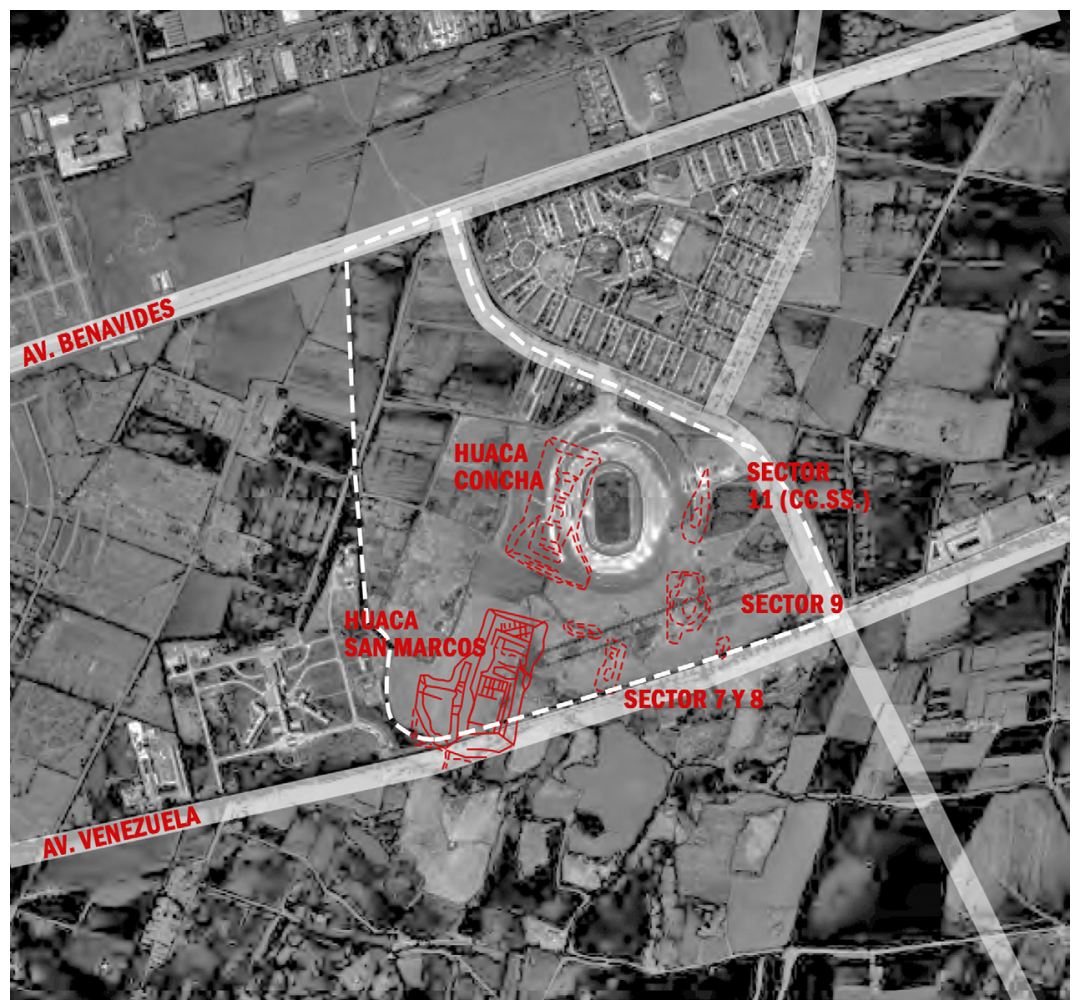

planificación de sus respectivos campus. Los planes urbanísticos o anteproyectos se desplegaron con eficiencia, a cargo de las respectivas comisiones de planeamiento; empero, el manejo político de los presupuestos otorgados por el Estado para la construcción de los proyectos fue desordenado y sin miras hacia un desarrollo a futuro. Ello dificultó la continuidad de un crecimiento orgánico, lo cual se ve reflejado hasta el día de hoy. Si bien los tres casos de estudio nacieron como proyectos de modernización nacional de influencia norteamericana — siguiendo la lógica de «ciudades a escala reducida» alejadas de la ciudad caótica-, presentan planteamientos distintos entre sí. Para el presente estudio de las ciudades universitarias se ha propuesto un análisis comparativo entre el anteproyecto -lo utópico — y lo construido —lo real—.

\section{Universidad Nacional Mayor de San Marcos: ciudad universitaria dispersa}

El anteproyecto, diseñado en 1957 por los arquitectos Alfredo Dammert, Carlos Morales Macchiavelo, Gerardo Lecca y Luis Dorich, enfrentó el reto de proyectar una ciudad universitaria en un terreno con preexistencias significativas: un estadio inconcluso y parte del conjunto arqueológico de $\mathrm{Ma}$ ranga. ${ }^{9}$ Los arquitectos optaron por ubicar la ciudad universitaria en el extremo inferior del terreno, entre las avenidas Venezuela y Universitaria. El plan se estructura a partir de un «paseo de estudiantes» de aproximadamente 


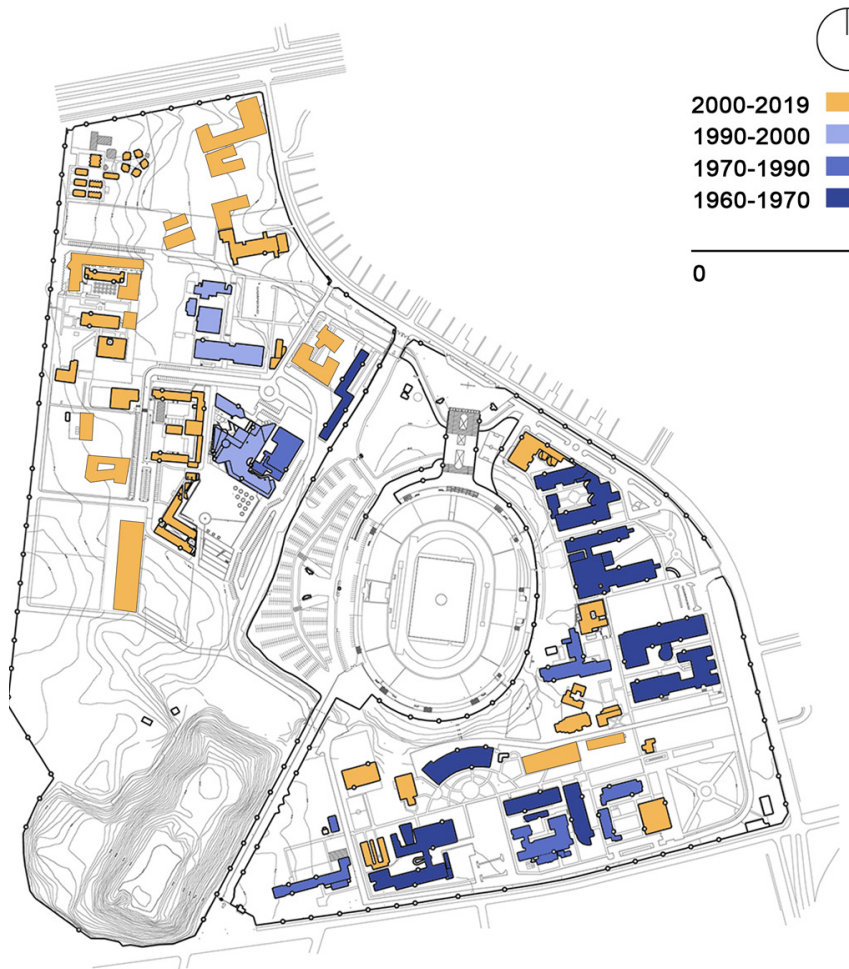

\section{FIGURA 6}

Arriba: evolución del campus de la UNMSM, desde 1960 hasta 2019. Abajo: diagnóstico urbano del campus actual. Elaboración propia, basada en el mapa de la Ciudad Universitaria (2013) proporcionado por la UNMSM.

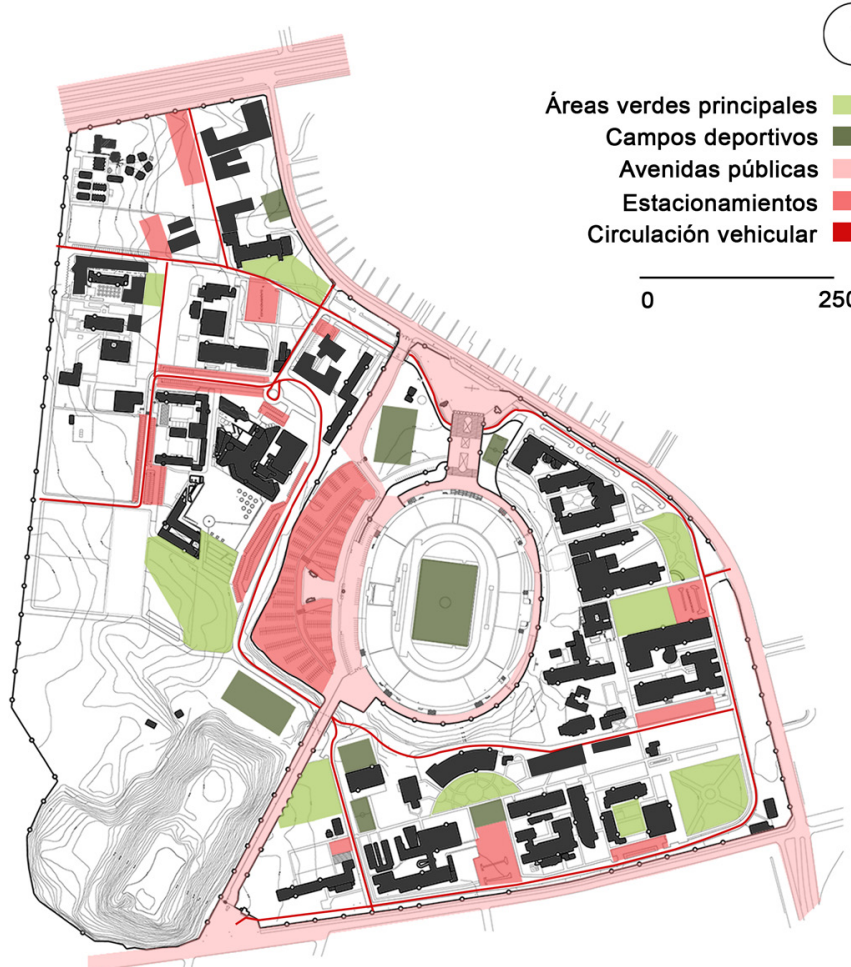


$50 \times 130$ metros, paralelo a la avenida Venezuela, que unifica cinco sectores diferenciados por funciones afines (Fabbri 2014: 388). El ingreso es hacia una gran explanada que agrupa los servicios comunes y la zona administrativa; atravesándola, se encontrarían las facultades organizadas a lo largo de la calle peatonal hasta culminar en la huaca San Marcos y un anfiteatro al aire libre. Al norte se encontraría la zona de viviendas para alumnos y docentes, integrada a un centro cívico.

En la década de 1960 se obtuvieron los primeros resultados; si bien la alameda se construyó, la ubicación de los pabellones varió. Los primeros edificios se ubicaron al sur del terreno: en paralelo a la alameda, la zona de ciencias naturales; y a la avenida Universitaria, la zona de humanidades. Los pabellones se diseñaron bajo premisas modernas: edificios longitudinales que rodean patios centrales, con rampas de acceso y recorridos continuos, separados de las zonas principales por grandes áreas verdes. Cada pabellón mantiene su autonomía; sin embargo, se conectan por medio de paseos peatonales y plazuelas, dejando la circulación vehicular en el perímetro.

Conforme la demanda estudiantil ha ido creciendo, la ciudad universitaria se ha expandido hacia el norte del terreno, conformando la nueva zona administrativa y central, así como la zona de estudios de posgrado. Lamentablemente, la organización del campus no se planificó en conjunto, de manera que los edificios se han añadido aleatoriamente en los espacios restantes. La inexistencia de un plan integrador resultó en una disociación entre el norte y el sur, a causa de la ubicación del estadio en el centro de la ciudad universitaria (figuras 5 y 6 ). ${ }^{10}$

\section{Universidad Nacional de Ingeniería: islas funcionales disgregadas}

10 Según Mario Meza, «El Estadio San Marcos aún se veía como un espacio aislado, no integrado, inutilizado y desperdiciado dentro de la ciudad universitaria [...] [L]a realidad del campus, porque no se había ajustado a un riguroso plan de crecimiento, obligó entonces a crear accesos al estadio por las avenidas Venezuela y Amezaga, dividiendo en dos la ciudad universitaria» (2009: 271).

11 Los centros arqueológicos ubicados en el sector de la Loma de Azúcar (UNI), huaca UNI-CISMID y huaca UNI-MINAS, pertenecen al Intermedio Tardío (11001440). Actualmente se mantienen como parte del campus, sin embargo, se encuentran en malas condiciones. El único montículo destruido es el que se encontraba en la Facultad de Arquitectura (Guzmán 2015: 104-126).
El primer anteproyecto data de 1944, a cargo de la firma Moore \& Hutchins (Nueva York), junto con los arquitectos Roberto Haaker Fort, Ricardo Valencia y Ricardo Malachowski. El plan se proyectó en la zona sur del terreno, en unión al Pabellón Central, edificio diseñado por Haaker Fort que se encontraba ya en plena construcción, a cargo de la firma Vargas Prada y Payet. Según la ficha del anteproyecto presentado en la revista El Arquitecto Peruano, el plan tampoco fue diseñado para ocupar la totalidad del terreno, ya que «las distancias habrían resultado demasiado grandes para un funcionamiento eficiente» (n. $\left.{ }^{\circ} 82,1944\right)$. Ello implicó que no tuvieran que enfrentarse a la preexistencia de restos arqueológicos, ya que las huacas se encontraban al norte del terreno. ${ }^{11}$ En menos de la mitad de la parcela se diseñaron dos pabellones: uno sería la ampliación del Pabellón Central, llamado Pabellón de Estudios Elementales; y el segundo, el Pabellón de Estudios Avanzados. El primero agruparía a los alumnos de primer y segundo año, la administración y la biblioteca, mientras que el segundo integraría los estudios especializados, como Ingeniería Mecánica, Ingeniería Civil, Arquitectura, Química y Minería. Estos dos edificios se complementarían con las residencias y servicios estudiantiles, como el campo deportivo, el centro estudiantil, un restaurante y el auditorio. La circulación vehicular sería perimetral y atravesaría el centro del proyecto siguiendo el eje del ingreso principal. 


\section{FIGURA 7}

Foto aérea, editada, con la ubicación de los restos prehispánicos de la UNI. Fuente: Guzmán 2015.

\section{FIGURA 8}

Izquierda: evolución del campus de la UNI desde 1945 hasta la actualidad. Derecha: diagnóstico urbano del campus actual de la UNI. Elaboración propia basada en el mapa de la Ciudad Universitaria (2004) proporcionado por la UNI.
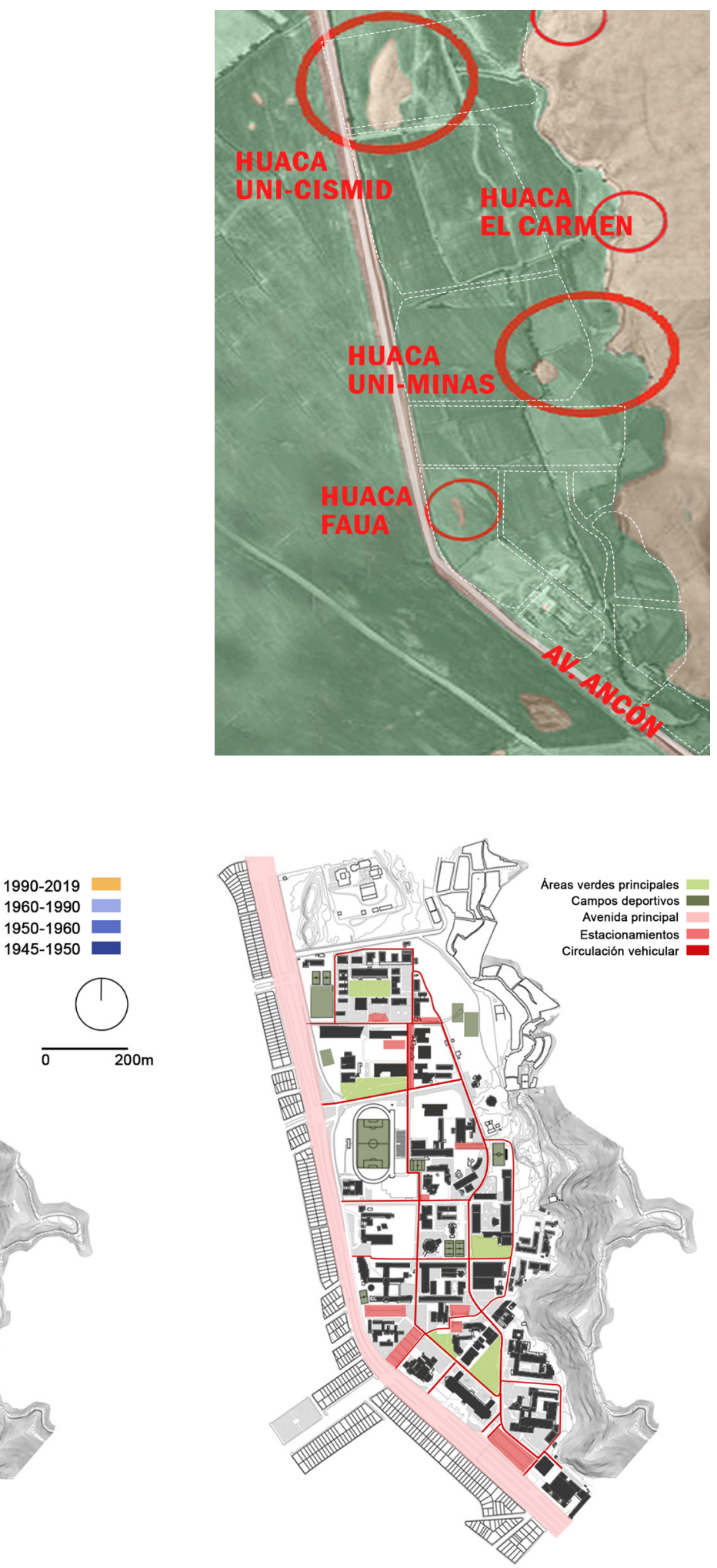


\section{FIGURA 9}

Fotografía panorámica, editada, de la Escuela Nacional de Agricultura en el fundo La Molina, década de 1940. Se observan cuatro pabellones de estudio, el rectorado y el internado, diseñados por el arquitecto Claudio Sahut en 1933. Fuente: Orlando Olcese (2002), 100 años de historia de la Universidad Nacional Agraria La Molina.

12 Las beautiful cities influyeron en el diseño: grandes vías rodeadas por jardines y sólidos pabellones, de la dimensión del Pabellón Central, y ausencia de vías peatonales entre edificios; mientras que las ciudades satélites influyeron en el emplazamiento alejado del núcleo urbano (OCPLA-UNI 2004: 42).

\section{FIGURA 10}

Arriba: evolución del campus de la UNALM desde 1933 hasta la actualidad. Abajo: diagnóstico urbano del campus actual de la UNALM. Elaboración propia, basado en el mapa de la Ciudad Universitaria (1983) proporcionado por la UNALM.

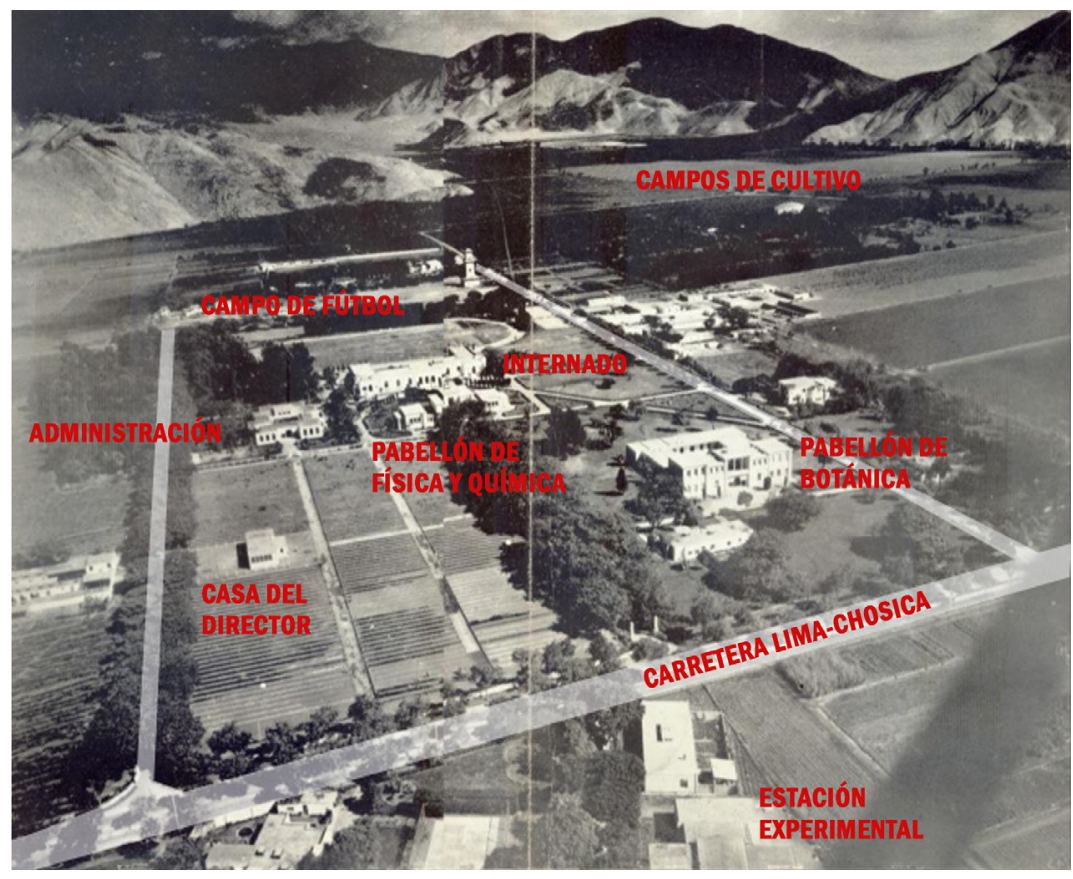

No obstante, el plan parece no haber tenido relevancia alguna, ya que el segundo edificio que se construyó fue el Pabellón de Petróleo, en 1947, que no pertenecía al anteproyecto. Salvo por la circulación vehicular, todos los demás criterios fueron descartados. La década de 1950 significó la inauguración oficial del campus universitario, ya que se terminaron de construir los pabellones de las facultades de Arquitectura (1953), Ingeniería Civil (1955-1957), Ingeniería Química e Industrial (1952) e Ingeniería Mecánica y Electricidad (1955); esto, acompañado de veredas y pistas, además de otros laboratorios adyacentes a los pabellones (López Soria 2003: 87). Debido a que el proyecto inicial se formuló bajo los postulados de las beautiful cities norteamericanas y las ciudades satélites inglesas, el resultado fue la creación de islas independientes agrupadas por funciones específicas y desarticuladas entre sí. ${ }^{12}$ Por otra parte, al carecer de un proyecto urbano que abarcara el terreno en su totalidad, no se diseñó el espacio público; se dejaron áreas verdes en los espacios residuales entre los pabellones y las vías de circulación. Tampoco hay plazas de concentración diseñadas para tal fin; y las pocas existentes no se diseñaron con un uso específico, por lo que terminaron siendo espacios difusos. Por último, los servicios comunes — como el comedor estudiantil, el gimnasio o el teatro- no poseen una ubicación adecuada en relación con los edificios académicos (figuras 7 y 8).

\section{Universidad Nacional Agraria La Molina: utopía construida paralizada}

El anteproyecto de la UNALM se dio más tardíamente que los otros casos de estudio. En 1961 se creó una comisión compuesta por arquitectos 

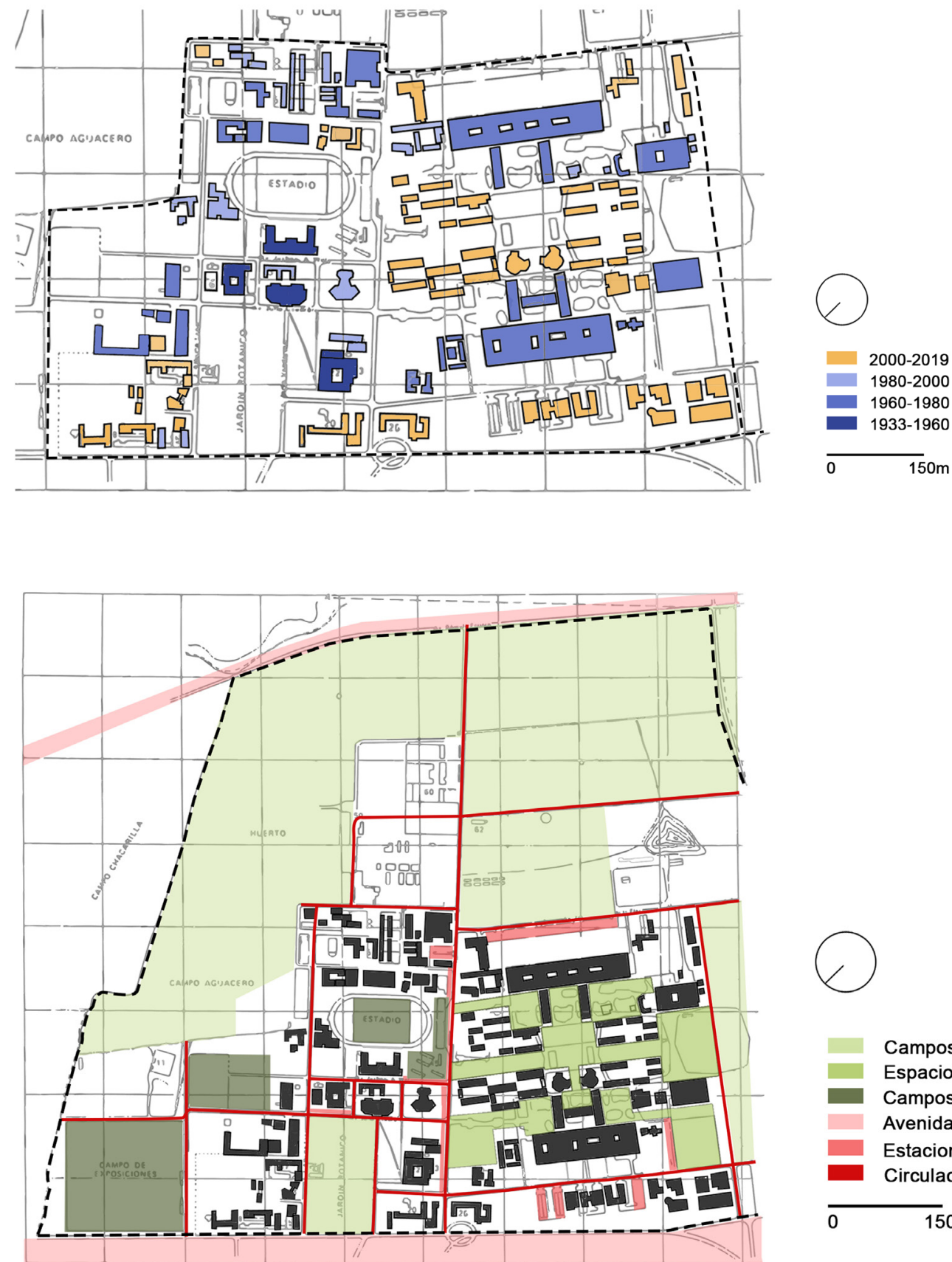

Campos de cultivo Espacios comunes Campos deportivos Avenidas principales Estacionamientos Circulación vehicular

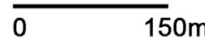


norteamericanos y especialistas nacionales: Edward Waugh, Robert Eheredge, Enrique Rivero y Salomón Martínez, entre otros. Posterior al terremoto de 1940, una de las preocupaciones principales fue el estudio de suelos, así como un diseño antisísmico para los edificios. El plan se adecuó, entonces, a las edificaciones que se encontraban todavía en pie luego del desastre: la administración, el Pabellón de Física y Química y el Pabellón de Botánica, edificios diseñados por el arquitecto Claudio Sahut en 1933. El plan posee una avenida principal vehicular, López de Romaña, que estructura el proyecto creando dos sectores bien diferenciados: la zona administrativa y de servicios comunes hacia el este, y la zona de estudios — con las facultades y los laboratorios - hacia el oeste. En el primer caso la parcela está dividida por la circulación vehicular, mientras que en el segundo se remite al perímetro de la agrupación de facultades, a la cual solo se puede acceder peatonalmente. Los campos de cultivo y de experimentación se encuentran en el perímetro del terreno y al frente de la avenida La Universidad. Es importante destacar la composición de grandes áreas verdes junto a la gran plaza de concentración entre las facultades. Asimismo, se buscó la horizontalidad de los edificios para lograr una integración orgánica con el área abierta del contorno.

Las obras se iniciaron en los primeros años de la década de 1960: tanto los edificios de las facultades — que agrupaban Economía y Planificación, Agronomía, Ciencias, Industrias Alimentarias, entre otras- como el de la Biblioteca Central, de gran prioridad. A diferencia de los casos de estudio previos, la obra y el proyecto urbano sí tuvieron correspondencia. En 1968 se inauguró la primera etapa de la ciudad universitaria, con dos facultades y los laboratorios de Ciencias e Ingeniería Agrícola (Olcese 2002: 476). Mientras que los pabellones eran bloques en barra de tres pisos, las aulas y los laboratorios mantenían una escala reducida, de bloques horizontales de un piso con estructuras techadas irregulares. Sin embargo, luego del terremoto de 1974 las aulas fueron destruidas totalmente y reemplazadas, durante años, por estructuras prefabricadas: recién en 2009 se terminaron de construir en su totalidad, nuevamente de concreto. Lamentablemente, se perdió el lenguaje arquitectónico moderno; en la actualidad son bloques idénticos, cerrados y sin intención urbanística.

A fines de 1970, debido a la inseguridad y los constantes robos, se cercó el terreno de la UNALM; y por la presencia de movimientos políticos extremistas, se eliminaron las viviendas al interior del campus, tanto de obreros como de estudiantes (Olcese 2002: 618). El resultado es una demostración de que no hay plan urbano que no deba modificarse en el tiempo. Si bien los primeros planteamientos eligieron como opción ideal el modelo de la ciudad universitaria norteamericana moderna, en la actualidad muchos de sus principios han perdido vigencia, lo que evidencia una carencia de planificación a futuro, como ciudad universitaria (figuras 9 y 10).

\section{Reflexiones finales}

Los tres casos de estudio, por su magnitud territorial e influencia en la idiosincrasia de la sociedad, están considerados como las ciudades universitarias de 
mayor relevancia en Lima durante el siglo XX. El impulso de modernizarse como instituciones públicas nacionales ante la demanda estudiantil, más el anhelo de consolidarse como universidades modernas, a semejanza de otras reconocidas universidades latinoamericanas, hizo posible la lucha contra el desinterés y el desamparo económico del Estado, así como la crisis política tanto universitaria como nacional.

Las influencias extranjeras fueron un impulso para académicos que no solo se enfrentaron al retraso en la modernización, sino que también se anticiparon al crecimiento de la ciudad y la contención de grandes masas estudiantiles. El emplazamiento de los campus, si bien tuvo lugar en las afueras de la trama urbana, bajo premisas norteamericanas - la segregación del campus respecto a la ciudad, rodeado de áreas verdes y zonificado por funciones específicas-, terminó por incentivar el crecimiento y la expansión de la ciudad hacia las periferias. En la actualidad nos enfrentamos a islas universitarias ubicadas alrededor de tramas urbanas ya consolidadas a las que — más allá de integrarse a ellas - les dan la espalda a través de muros perimétricos. En paralelo, la ciudad se ha expandido a tal punto que las universidades nuevas optan por segmentarse en facultades esparcidas por la ciudad. La implantación de una ciudad universitaria o de un edificio universitario no plantea disyuntivas decisivas y su importancia no reside en la elección de uno u otro, sino en cómo se interrelaciona con la trama urbana adyacente, en pos de mejorar las condiciones de vida del estudiantado y de los ciudadanos en general. Es entonces prudente plantearnos cuestionarnos: ¿qué beneficios resultan de una ciudad universitaria sin muros? ¿Cómo conseguir esa reconciliación entre universidad y ciudad? Es una tarea pendiente y necesaria.

\section{Bibliografía citada}

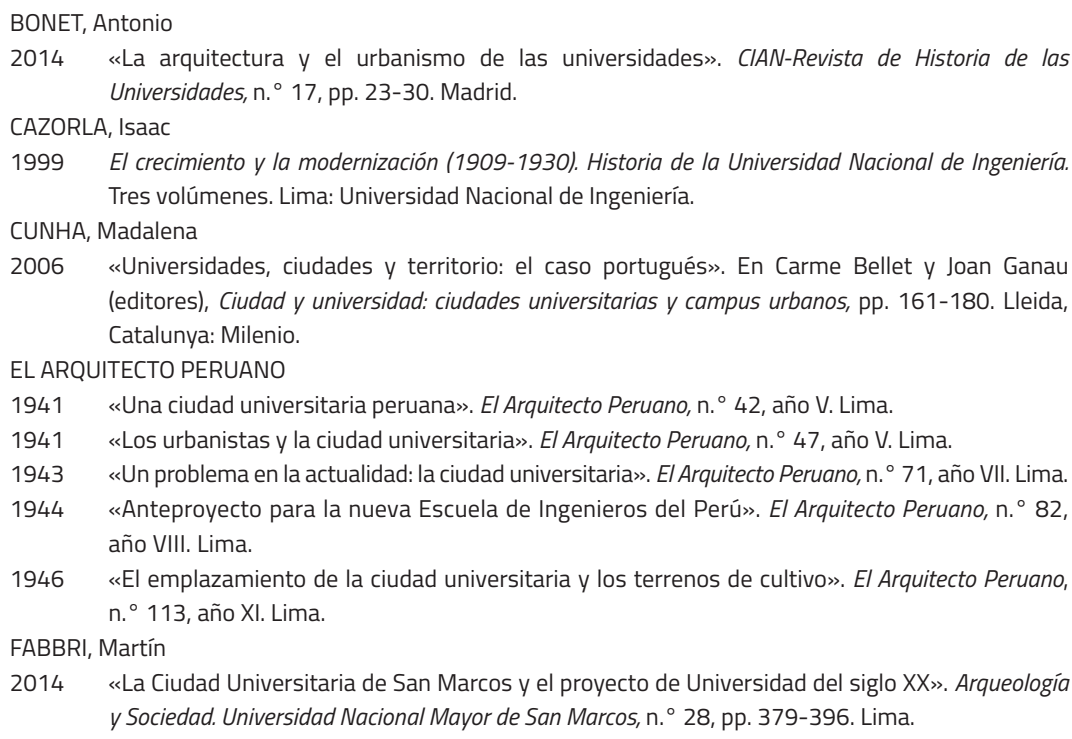

2014 «La Ciudad Universitaria de San Marcos y el proyecto de Universidad del siglo XX». Arqueología y Sociedad. Universidad Nacional Mayor de San Marcos, n. ${ }^{\circ}$ 28, pp. 379-396. Lima. 
FINO GÓMEZ, Carlos

2018 «Elementos conceptuales de las ciudades universitarias en América Latina para la consolidación y conservación del Campus Bogotá de la Universidad Nacional de Colombia». En Ciudades universitarias: un proyecto moderno para América Latina, pp. 14-47. Bogotá: Universidad Nacional de Colombia

FLORES, Alfonso

2000 «Contribución a la historia de la Universidad Nacional Agraria La Molina». En Construyendo el Perú: aportes de ingenieros y arquitectos, pp. 167-186. Lima: Universidad de Ingeniería, Proyecto Historia UNI.

GARFIAS, Marcos

2009 «La formación de la universidad moderna en el Perú: San Marcos, 1850-1919». Tesis de licenciatura en Historia presentada a la Universidad Nacional Mayor de San Marcos.

GUTIÉRREZ, Ramón

1983 Arquitectura y urbanismo en Iberoamérica. Madrid: Cátedra.

GUTIÉRREZ, Ramón y Rodrigo GUTIÉRREZ

2012 «Una mirada crítica a la arquitectura latinoamericana del siglo XX. De las realidades a los desafíos». En Enrik Karge (editor), 1810-1910-2010. Independencias dependientes. Art and national identities in Latin America. Dresde: Universidad de Dresde.

GUZMÁN, Enrique

2015 «Huacas de la Universidad Nacional de Ingeniería y la verdadera Huaca Aliaga». Devenir, vol. 2, n. ${ }^{\circ}$ 4, pp. 104-126. Lima.

LÓPEZ SORIA, José

2005 UNI. De Escuela a Universidad. Historia de la Universidad Nacional de Ingeniería. Tres volúmenes. Lima: Universidad Nacional de Ingeniería.

LUDEÑA, Wiley

2001 «Fernando Belaúnde Terry y los inicios del urbanismo moderno en el Perú». En Construyendo el Perú Il: Aportes de ingenieros y arquitectos. Lima: Proyecto Historia UNI, pp. 245-286.

MERLIN, Pierre

2006 «Campus o regreso a la ciudad? Las relaciones espaciales ciudad-universidad». En Carme Bellet y Joan Ganau (editores), Ciudad y universidad: ciudades universitarias y campus urbanos, pp. 183-201. Lleida, Catalunya: Milenio.

MEZA, Mario

2009 «Historia del Estadio de la Universidad Nacional Mayor de San Marcos». Cuadernos del Instituto Antonio de Nebrija, n. ${ }^{\circ} 12$, pp. 243-282.

OFICINA CENTRAL DE PLANIFICACIÓN (OCPLA -UNI)

2004 Diagnóstico físico espacial de la Universidad Nacional de Ingeniería - Espacios colectivos. Lima: Universidad Nacional de Ingeniería.

OLCESE, Orlando

2002 Enfrentando la adversidad, camino a la gloria: Historia de la Universidad Nacional Agraria La Molina (1902-2002). Lima: Universidad Nacional Agraria La Molina

RODRÍGUEZ, Katya

1999 La apertura a espacios nuevos (1930-1955). Historia de la Universidad Nacional de Ingeniería. Tres volúmenes. Lima: Universidad Nacional de Ingeniería.

YEPES, Ernesto

1986 «La Escuela Nacional de Agricultura». En Estudios de historia de la ciencia en el Perú, volumen 1, Ciencias Básicas y Tecnológicas, pp. 193-213. Lima: Sociedad Peruana de Historia de la Ciencia y la Tecnología. 